\title{
Use of Optoelectronic Systems for the Analysis of Technique in Trials
}

\author{
Rodolfo Vastola, Vladimir Medved, Albano Daniele, Silvia Coppola and Maurizio Sibilio \\ Motion Analysis Laboratory, Department of Human, Philosophical and Educational Sciences, University of Salerno, Fisciano \\ Salerno 84084, Italy
}

\begin{abstract}
Optoelectronic systems represent the gold standard of technologies used in the motion analysis for the evaluation of kinematics. Trials, a specialty of mountain biking, differ a lot from other cycling specialties, because in addition to pedaling, they require jumping techniques to overcome obstacles that involve the whole body. In this sport, more than in others, the evaluation of technical movements using optoelectronic systems goes far beyond simply assessing the biomechanics of pedaling or the correct positioning on the bike. Starting from a previous study conducted with video analysis technique applied to the lateral jumping technique, two versions of this technique were compared, considering the kinematics of the act, taking into account both the data relating the subject and the bicycle. The joint angles of the lower limbs and the angles described by the bicycle on the three space plane were considered. The major differences have been found in the "leap over" phase, about the bending of the lower limbs and the angles described by the bicycle.
\end{abstract}

Key words: Optoelectronic systems, trials, kinematics, side hop technique.

\section{Introduction}

Trials are a specialty of off-road cycling in which it is required to overcome short obstacle courses without putting the feet on the ground. In the U.C.I. (International Cycling Union) rules, only bicycle tires can touch the ground and obstacles, otherwise penalties are assigned [1]. In competitions, there are multiple obstacle courses that need to be completed for a number of times. The athlete has about two minutes to complete the course, if he exceeds the time limit, penalties are assigned. On each course, more routes are tracked and they are marked with different colors corresponding to the different categories. The type of obstacle varies greatly, some competitions are conducted in natural environments using rocks in the course of rivers or mountains, in other cases, artificial obstacles are used like big tires, trunks or concrete obstacles. The obstacles' height ranges from $50 \mathrm{~cm}$ to

Corresponding author: Rodolfo Vastola, Ph.D., assistant professor, research fields: sports and motor performance analysis. over $2 \mathrm{~m}$. In the practice of Trials special bikes are used that need to combine strength and lightness. Unlike the other specialties of cycling, in Trials it is not required to ride for long distances, obstacles are mostly overcome with a jump technique in which the whole body is involved. Among the specialties of cycling, this is definitely one where technique plays a predominant role. A good riding technique allows the athlete to overcome the obstacles in an optimal manner with a minimal effort and reduces the possibility of committing penalties. It is precisely in the evaluation of technical movements that technologies such as optoelectronic systems find their place [2]. Optoelectronic systems represent the gold standard for the detection of kinematic parameters, displacement, velocity, acceleration, and joint angles measurement. An optical telemetry [3] system consists of a series of special cameras that can capture the infrared light and by special markers that are applied on the subject under examination. Usually, to reconstruct a three-dimensional point, an optical telemetry system needs a minimum of two cameras placed at known 
distances from each other; in this way, thanks to triangulation operations, it is possible to go back to the actual position of a point in space with millimeter accuracy in modern systems. The most common optical systems use LEDs of infrared light emitters placed in a corolla around the lens of the camera and a series of reflecting markers. In this work the side hop [4], technique was taken into account, a technique that allows the athlete to climb up an obstacle by side and perform a jump with a loading phase, like a counter-movement squat jump. Within this technique, there are many versions of finalizing phase, depending on the type of obstacle to be overcome. Here the two most used versions were compared: one involves contact of the front wheel first, using pivot action to bring the rear wheel over the obstacle; the other involves the complete cross of the obstacle and is used especially when it is not possible to use the obstacle as an aid to perform the "front pivot", as in the case of a jump on a bar. To compare the two types of jump, a series of jumps were performed both on a cube of $1 \mathrm{~m}$ in height and on a bar placed at $1 \mathrm{~m}$ from the ground. The tests were taken with the aid of an optical telemetry system consisting of six cameras and passive markers. A force platform was also used. To our knowledge there are currently no studies that use optoelectronic systems for the analysis of technique in the Trials.

\section{Objectives}

This study has as its first objective the introduction of the motion analysis technologies, such as optoelectronic systems, in the sport of Trials, and the development of data acquisition and data processing protocols, which can be used for future investigations in this sport. Secondly, to operate an analysis on two different executions of the side hop technique, a comparison was already made in a previous study conducted with the video analysis technique. In this way the data provided by quantitative analysis will complement those of qualitative nature of the earlier work, to provide coaches with useful information for the improvement of performance.

\section{Methodology}

\subsection{CAST Protocol}

The CAST (calibrated anatomical system technique) protocol [5], with the application of cluster of markers, is a technique that is based on the principle that, considering a rigid body, and establishing a local reference system integral to this, the location of all the points belonging to the body does not vary, with respect to this reference, in time. In this way, given a reference system fixed to the bone segment in question, constituted precisely by a cluster of at least 3 markers not aligned, and proceeding with a first static acquisition of the points taken into consideration, it is possible to subsequently remove the markers, leaving only the cluster during dynamic acquisition, and then reconstruct through software the location of all the other points at every moment of the performance.

\subsection{Davis Protocol}

The Gait Analysis procedure used in this study is the Davis Protocol which starts with measurement of the subject's anthropometric parameters such as weight, height, tibial length, distance between the femoral condyles or diameter of the knee, distance between the malleoli or diameter of the ankle, distance between the anterior iliac spines and thickness of pelvis.

The protocol uses 20 markers, respectively C7, acromion, A.S.I.S. (anterior superior iliac spine), sacrum, trochanters, the middle of thigh, lateral condyle, fibular head, the middle of leg, lateral malleolus, the 5th metatarsal head [6]. During static capture two markers are used on the heels to calculate the angle of offset.

\subsection{Subject}

The participant in this study was a 27 years old male, $1.82 \mathrm{~m}$ height and $77 \mathrm{~kg}$ of body weight, right foot dominant, with 8 years of experience in Trials riding. 
In Trials, the dominant foot refers to the foot which is kept forward when riding the bike.

\subsection{Instruments}

To obtain data relating to the kinematics, a BTS Smart-D Optoelectronic system was used consisting of six cameras with an acquisition frequency of up to 140 $\mathrm{Hz}$ mounted on adjustable stands and stickers reflective markers. A BTS P6000 force platform was used for the identification of the first contact in the loading phase, however the data for the dynamic aspects of the jump have been neglected since some variables, such as compression of the rubber and the return of elastic energy, would distort the measurement of the detected force, not being the survey directed to the measurement of these parameters, it was preferred to use only the information about the contact and detachment of the wheel from the platform. To perform the tests the Echo MkII 26 bicycle was used, characterized by geometry 1080/+55/380/72 ${ }^{\circ}$ (respectively wheelbase length, bottom bracket height from the wheelbase line, chainstay length and head tube angle), mechanical disc brake on the front and rear hydraulic rim brake. The obstacles used for performing the jumps were a cube of $1 \mathrm{~m}$ in height and a bar mounted on two metal uprights at $1 \mathrm{~m}$ (similar to the bar of the high jump used in athletics).

\subsection{Procedure}

The environment was prepared in an optimal manner

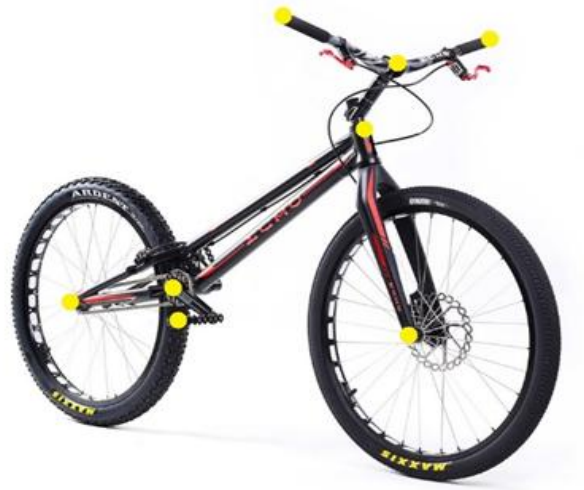

(A) for the analysis to be carried out. The center of the workspace consisted of force platforms, embedded within a platform used for the gait analysis, all the way around the six cameras of the telemetry system have been arranged. A calibration of the optoelectronic system was carried out on a three axes reference frame positioned at the center of the acquisition volume. This operation had the purpose of making the position of the cameras with respect to the reference known to the system. The latter was set at a $70 \mathrm{~Hz}$ frequency of acquisition, sufficient to follow the movement without losing image quality. Later the markers [8] were applied. The markers used were of the passive type, beads covered with the infrared light reflecting material, applicable by adhesive. The points of application chosen on the bicycle were the left and right ends of both hubs (4), left and right ends of the bottom bracket (2), the center of the right and left pedals (2), the center of the upper head tube (2), the center of the handlebar and ends (3), for a total of 13 points (see Fig. 1).

The obstacle was marked with 6 markers, 3 to identify the upper floor, the other 3 on the sides. For the bar only 2 markers have been used, placed at the ends. The clusters used on the subject consisted of 3 markers, placed on a rigid support, that have been applied in five points, two on the thighs, two on the legs and one on the rear of the pelvis, at the sacral area, so as to determine, respectively, the position of the lower limbs and pelvis. Other markers were positioned on the

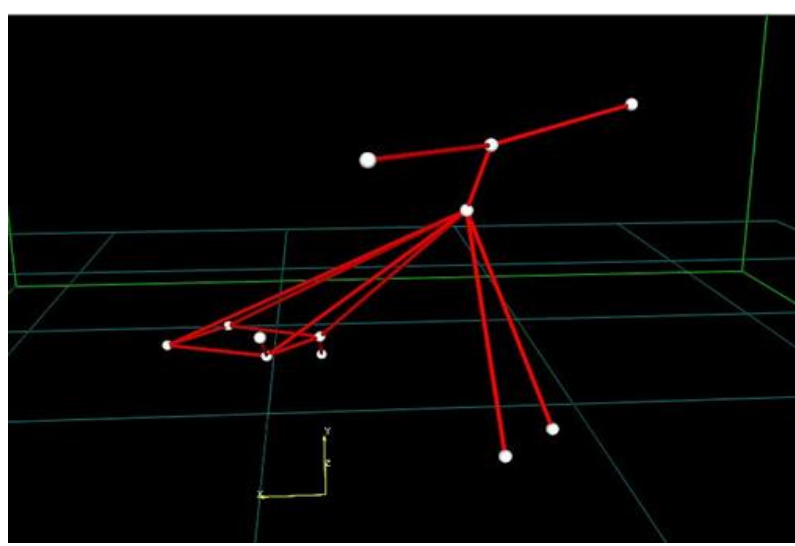

(B)

Fig. 1 (A) Yellow dots indicate the position of the markers; (B) the 3D model of the bike. 
subject according to the Davis protocol, only on the lower limbs, while the upper part of the body has not been taken into account. The points of application of the individual markers were, for the thigh, the trochanter and the condyle of the femur, for the leg, the head of the fibula and the ankle bone, for the pelvis, the anterior superior iliac spines and the sacrum, for the foot fifth metatarsal and heel (see Fig. 2) [7]. In total, 33 markers have been used for the subject. In order to apply the Davis protocol anthropometric measurements of the subject were previously provided.

A static acquisition of the bike was initially carried out, then, during the execution of the tests, the marker positioned on the right end of the bottom bracket was eliminated as it constituted a nuisance to the subject. The frame being a rigid body and having at least three points to define a local reference system, it was possible to reconstruct the marker in the post-acquisition thanks to dedicated software. A static acquisition of the subject was carried out following the CAST protocol, necessary for the reconstruction of the markers in the post-acquisition. Subsequently, all markers were removed from the subject, except for the clusters and for those related to the feet (5th metatarsal), thereby the work load was lightened for the system, which had to follow 19 markers instead of 33, and the subject was made free to move around without having to care about the markers. Also the obstacle and the bar were acquired, in particular a static acquisition of the obstacle has been carried out, in order to eliminate at a later time the markers positioned on the upper floor, so as not to be a hindrance to the subject, and to decrease the marker number to be acquired. These were then reconstructed in post-acquisition. For both jumps, seven tests were acquired, carried out consecutively. Rear tire pressure was measured before the tests $(1.7$ bar).

\subsection{Data Processing}

After the acquisition phase, the different tests have been provided by the BTS Smart-Analyzer software.
Thanks to the tracking operation it was also possible to obtain a 3-D model, and in addition the markers eliminated during dynamic acquisitions were reconstructed (see Fig. 3). All tests were then processed with the processing protocols, created specifically for this type of analysis, with which it was possible to get all the kinematic and dynamic data relating to the events examined. The application of the Davis protocol allowed the reconstruction of the joint centers and the collection of data relating to the joint angles of the lower limbs. For convenience, it was chosen to limit the results only to the loading and leap over phases, which were the most significant for comparing the two jumps. It was chosen to consider the beginning of the load as the opening phase of the cycle, and as the end time the leap over. The loading in both tests was made to coincide with the contact on the platform, the leap over, in the jump with the obstacle, has been identified as the point at which both of the hub center reach the same height, while in the jump with the bar as the highest point reached from the center of the rear hub.

\section{Results}

As already mentioned, for convenience the analysis was restricted to the time interval between the start of loading, identified as first contact registered by the force platform, and the time of leap over, which was

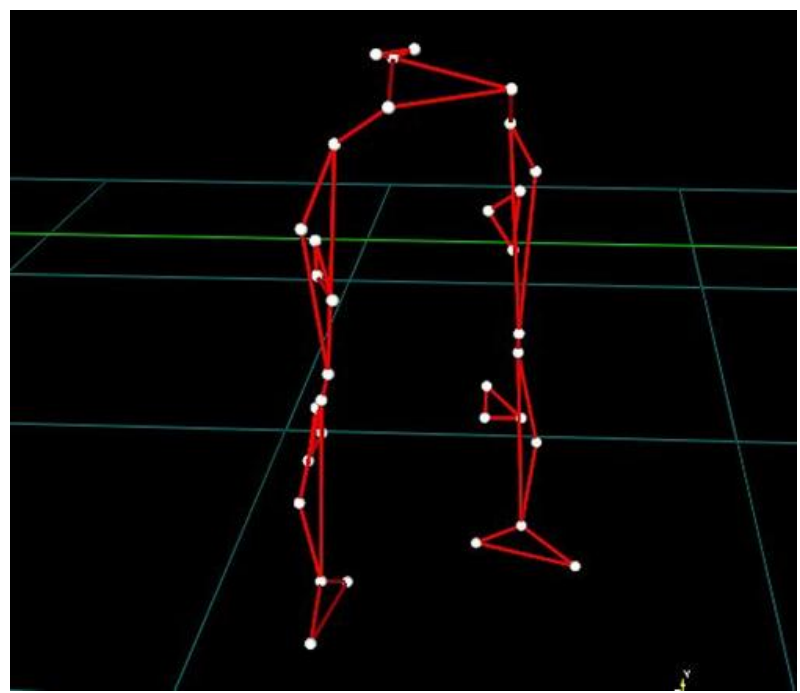

Fig. 2 A 3D model of the subject lower limbs. 


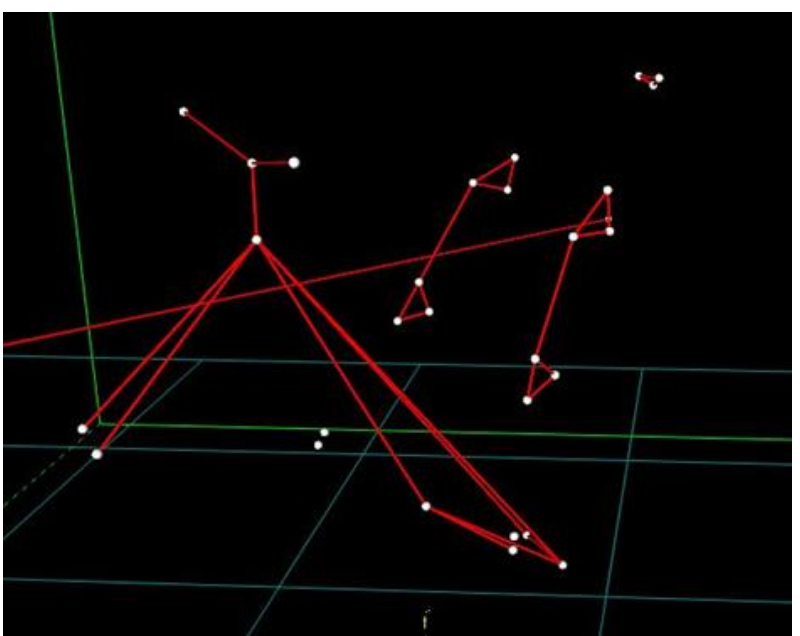

(A)

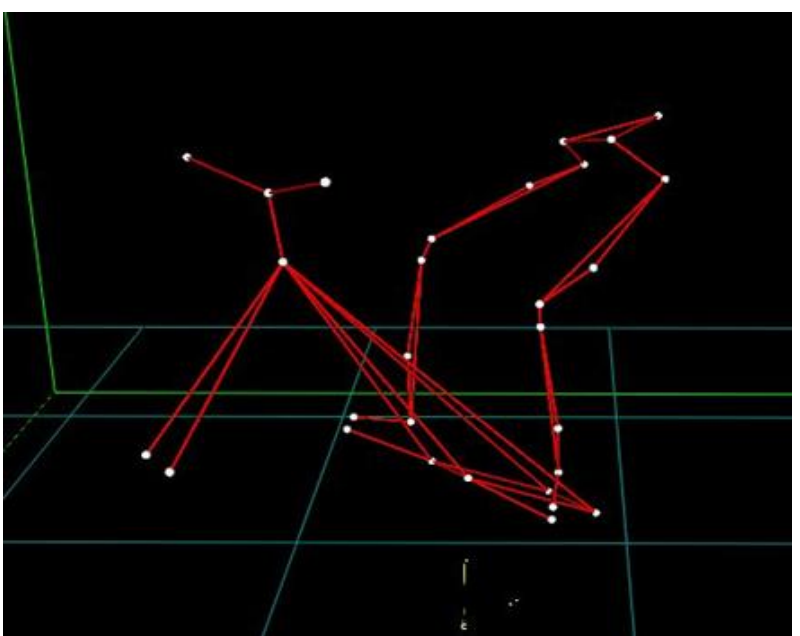

(B)

Fig. 3 Jump 3D model. (A) clusters; (B) final 3D model reconstruction.

Table 1 Angles described by bike in the bar jump.

\begin{tabular}{llll}
\hline & Frontalplane & Transversalplane & Sagittalplane \\
\hline Loading & $2.4(0.4)$ & $0.1(0.4)$ & $-40.1(1.3)$ \\
Take off & $5.6(2.2)$ & $-9.5(0.4)$ & $-55.9(2.1)$ \\
Leap over & $2.8(1.8)$ & $-1.5(4.2)$ & $-23.5(4.4)$ \\
\hline
\end{tabular}

Table 2 Angles described by bike in the obstacle jump.

\begin{tabular}{llll}
\hline & Frontalplane & Transversalplane & Sagittalplane \\
\hline Loading & $1.3(0.3)$ & $0.4(0.3)$ & $-40.9(1.4)$ \\
Take off & $3.1(2)$ & $-6.9(0.5)$ & $-53.8(2.2)$ \\
Leap over & $-4.9(2.8)$ & $-0.5(2.7)$ & $-4(2.3)$ \\
\hline
\end{tabular}

Table 3 Lower limbs flex-extension angles in the bar jump. Positive values for flexion; negative values for extension.

\begin{tabular}{lllllll}
\hline & \multicolumn{2}{c}{ Hip } & \multicolumn{2}{c}{ Knee } & \multicolumn{2}{c}{ Ankle } \\
\cline { 2 - 7 } & DX & SX & DX & SX & DX & SX \\
\hline Loading $\left[{ }^{\circ}\right]$ & $88.5(2.9)$ & $69.5(3.1)$ & $46.8(3.5)$ & $54.9(4)$ & $0.5(2.6)$ & $-2.4(3.1)$ \\
Take off $\left[^{\circ}\right]$ & $33.7(4.3)$ & $21.6(2.1)$ & $22.7(3.8)$ & $36.3(3.8)$ & $-12.5(2.6)$ & $-15.5(7.6)$ \\
Leap over $\left[{ }^{\circ}\right]$ & $126.1(1.3)$ & $125.4(2.5)$ & $125.4(5.9)$ & $153(1.7)$ & $14.5(3.9)$ & $17.7(7.8)$ \\
\hline
\end{tabular}

Table 4 Lower limbs flex-extension angles in the obstacle jump. Positive values for flexion; negative values for extension.

\begin{tabular}{lllllll}
\hline & \multicolumn{2}{c}{ Hip } & \multicolumn{2}{c}{ Knee } & \multicolumn{2}{c}{ Ankle } \\
\cline { 2 - 7 } & DX & SX & DX & SX & DX & SX \\
\hline Loading $\left[{ }^{\circ}\right]$ & $87.8(3.1)$ & $67.8(3.2)$ & $44(3.4)$ & $49.6(2.3)$ & $0.3(2.8)$ & $1(2.5)$ \\
Take off $\left[{ }^{\circ}\right]$ & $46.8(3.2)$ & $28.4(3.7)$ & $22.7(3.8)$ & $28.7(6.2)$ & $-14.3(1.2)$ & $-14.8(6)$ \\
Leap over $\left[{ }^{\circ}\right]$ & $122.1(2.5)$ & $108(3.7)$ & $96.7(4.7)$ & $138.1(5.1)$ & $7.1(2)$ & $28.4(1.8)$ \\
\hline
\end{tabular}

Table 5 Loading time comparison.

\begin{tabular}{ll}
\hline Loading time (obstacle) [ms] & $442.86(24)$ \\
Loading time (bar) [ms] & $484.19(29)$
\end{tabular}

Table 6 Loading depth comparison.

\begin{tabular}{ll}
\hline Min sacrum height(obstacle) $[\mathrm{m}]$ & $1.26(0.04)$ \\
Min sacrum height (bar) [m] & $1.21(0.04)$ \\
\hline
\end{tabular}


made to coincide with the maximum height reached by the rear axle. As to kinematic data, the lower limbs flexion-extension angles with respect to the subject were taken into consideration, while for the bike the rotations on all three axes were taken into account. The angular values were considered in three moments, the beginning of loading, take-off and leap over. The lowest point reached by the sacrum was also considered as indicating the depth of the load.

\section{Discussion and Conclusions}

Comparing the data obtained for both modes of jumping, clear differences emerge especially during leap over phase. The pivoting action of the front wheel is identified by observing the variations of the bike angle in sagittal plane at the time of leap over, here the biggest difference between the two execution methods is recorded. In jumping the obstacle, it tends to zero, with mean values of $4^{\circ}$, while in the other case the angle reaches about $23.5^{\circ}$. Another difference is found in the angle described in the frontal plane. In this case, the average values of $-4.9^{\circ}$ recorded in jumping obstacle, are due to the "search for" the obstacle during the pivot action on the front wheel, which then would lead to an inclination from the opposite side of the obstacle, a situation that does not absolutely occur in the other kind of jump, which has the opposite trend, however, though less obvious, with an average angle of $2.8^{\circ}$ which therefore indicate a slight inclination towards the obstacle. The loading times are slightly longer in the bar jump, probably due to a more accentuated loading also shown by a deeper descent of the pelvis average of $5 \mathrm{~cm}$. Substantial differences are found in the kinematics relative to the action of the lower limbs compared to the angles of flexion-extension of the hip, knee and ankle. In the initial stage of loading there is a good overlap between the tests, however, at the time of take-off a greater extension is recorded, even at the hips level, in the jump performed on the bar, because obviously in this case a greater verticality is searched, unlike the jumping obstacle, in which probably the smaller extension of the hip is due to the anticipatory action of the displacement towards the front wheel to look for the support on the obstacle to make the pivot action. However, the biggest difference can be found at the knee at the time of leap over. In the bar jump, there is a much greater flexion at the knee joint compared with the obstacle jump, as it is necessary to completely overcome the bar without touching it. At the level of the ankle, there is a big difference between the two versions. In the bar jump, ankles have roughly the same degree of flexion, in the obstacle jump the left is bent around $28^{\circ}$, while the right is only $7^{\circ}$, this in line with what was seen with respect to the position of the bicycle tilted to the left at the time of leap over.

To our knowledge, this work is the first in which optoelectronic systems are used for the analysis of technique in Trials, thus representing a first step for the introduction of this type of instruments in this sport, which is the main objective of this work. Acquisition and processing protocols specially conceived for this discipline were developed. In particular, the marker set on the bicycle and the use of clusters, a choice detected as optimal for the freedom of movement of the subject. This in line with the set objectives could be a starting point for further analysis in Trials using optoelectronic systems. As to the comparison of the two jumps, from the results we obtained it was possible to quantitatively identify the differences between the two modes. On the basis of these results, we can say that between the two jumping modes, the "front pivot" technique, that uses the obstacle as an aid, presents a more economic movement, with smaller joint excursions and a lesser displacement with equal height reached. This study could support coaches to understand which elements can most influence the success of a technique and how to improve the performance. Optimizing the front pivot action allows to reach greater heights with less energy expenditure. Also considering the fact that the bar jumping technique requires greater range of movement and thus presumably increased energy expenditure, it 
could be useful and more interesting to go deeper into the study of front pivot action to find a way to best develop this more efficient jump technique. On the other hand, the results of this study suggest that, to improve the bar jumping technique, coaches may optimize the joint mobility of athletes, because the flexion of the lower limbs is the element that most characterizes this technique. The data already acquired, even if not all referred to herein, together with the acquisition protocols and data processing, created to examine this pattern, undoubtedly constitute an important resource for future comparison between different athletes, or between different jump executions of the same athlete.

\section{References}

[1] U. C. I. 2015. International Cycling Union "Regulations, Part VII": Trials. Aigle: U. C. I. Accessed August 10, 2016.

http://www.uci.ch/mm/Document/News/Rulesandregulati on/16/91/28/7-Tria-E-versionon180315_English.pdf
[2] Cappozzo, A., Della Croce, U., Leardini, A., and Chiari, L. 2005. "Human Movement Analysis Using Stereophotogrammetry: Part 1: Theoretical Background." Gait \& Posture 21 (2): 186-96.

[3] Puglisi, F. 2007. Biomechanics: Introduction to Posture and Movement Instrumental Measures. Roma: Marrapese, 168-75. (in Italian).

[4] Happich, J. 2012. Your Complete Guide to Trials Riding. Parigi: Wild Grip Publishing, 164-77.

[5] Cappozzo, A., Cappello, A., Croce, U. D., and Pensalfini, F. 1997. "Surface-marker Cluster Design Criteria for 3-D Bone Movement Reconstruction." IEEE Trans. Biomed. Eng. 44 (12): 1165-74.

[6] Davis, R. B., Ounpuu, S., Tyburski, D., and Gage, J. R. 1991. "A Gait Analysis Data Collection and Reduction Technique." Human Movement Science 10 (5): 575-87.

[7] Cappozzo, A., Catini, F., Della Croce, U., and Leardini, A. 1995. "Position and Orientation in Space of Bones during Movement: Anatomical Frame Definition and Determination." Clinical Biomechanics 10: 171-8.

[8] Payton, C., and Bartlett, R., eds. 2007. "Biomechanical Evaluation of Movement in Sport and Exercise." In The British Association of Sport and Exercise Sciences Guide. Routledge. 